\title{
TRACKING CONTROL IN THE PRESENCE OF NONLINEAR DYNAMIC FRICTIONAL EFFECTS: ROBOT EXTENSION
}

\author{
M. Feemster, D.M. Dawson, A. Behal and W. Dixon
}

\begin{abstract}
In this paper, we extend the observer/control strategies previously published in [25] to an $n$-link, serially connected, direct drive, rigid link, revolute robot operating in the presence of nonlinear friction effects modeled by the LuGre model. In addition, we also present a new adaptive control technique for compensating for the nonlinear parameterizable Stribeck effects. Specifically, an adaptive observer/controller scheme is developed which contains a feedforward approximation of the Stribeck effects. This feedforward approximation is used in a composite controller/observer strategy which forces the average square integral of the position tracking error to an arbitrarily small value. Experimental results are included to illustrate the performance of the proposed controllers.
\end{abstract}

KeyWords: Friction compensation, nonlinear control.

\section{INTRODUCTION}

With the recent introduction of robot manipulator systems into such high precision applications as that of micro-surgery, researchers in the area of robotics are challenged to develop electromechanical systems that allow for the exact placement of robot end-effectors. That is, the mechanical design coupled with the precise control of joint actuators must promote accurate placement of the robot manipulator. However, the performance of model-based control strategies for these mechanical systems is ultimately limited by the model's ability to accurately describe the system dynamics. With the accuracy required in many position tracking or setpoint applications, the procurement of more accurate friction models and the corresponding model-based controllers for robot manipulator systems is apparent. Hence, several robotic researchers are currently focusing on the development of controllers based on more accurate/complex friction models in an attempt to increase the performance of robot manipulator systems.

Due to an extensive history of research on the friction phenomena, a plethora of control algorithms currently exist that employ numerous friction models ${ }^{1}$. For example in [8], Canudas et al. utilized a linear parameterizable friction model to develop an adaptive control algorithm to

Manuscript received September 28, 1999; accepted October 28, 1999 .

This work is supported in part by the U.S. National Science Foundation Grants DMI-9457967, CMS-9634796, ECS-9619785, DOE Grant DE-FG07-96ER14728, the Square D Corporation, and the Union Camp Corporation. promote link position tracking for a robot manipulator. Gonzalez et al. [14] utilized nonlinear feed-forward compensation for friction while obtaining force control in robotic manipulators. In [2], Armstrong explored the implications of the Stribeck effect on feedback control using a reduced order model of friction. Armstrong also established experimental procedures to stabilize the performance of the controller at low velocities. In [4], Armstrong applied dimensional and perturbation analysis to solve a nonlinear low-velocity friction control problem. Specifically, a simple PD controller was implemented after reducing the parameters representing the friction model from ten to five via a dimensional analysis. In [13], Friedland et al. proposed an adaptive control scheme which entails the use of an observer to estimate the kinetic friction coefficient. Annaswamy et al. [1] developed an adaptive tracking controller for a class of systems with convex/concave parametrization. The proposed controller of [1] can be directly applied to mechanical systems with significant friction effects to achieve globally uniformly ultimately bounded position tracking. In [12], Feemster $e t$ al. developed an adaptive controller that achieved global asymptotic setpoint regulation while actively compensating for the nonlinearly parameterizable Stribeck effects.

With the recent concern over the ability of static friction models to accurately capture such low velocity friction effects as hysteresis and stick-slip, researchers have focused on the development of alternative dynamic friction models. To address the shortcomings of static

\footnotetext{
${ }^{1}$ We refer the reader to [3,5,7] and [15] for reviews on the various friction modeling techniques.
} 
models, Canudas et al. [7] proposed a dynamic model for friction that more accurately predicts friction behavior caused by the Stribeck effect, hysteresis, and the varying break-away force. In addition, Canudas et al. [7] explored the development of observer-based control strategies for the proposed model. Olsson et al. [20] analyzed an observer-based control strategy for the dynamic friction model of [7] with regard to the controller's sensitivity to modeling errors and sensor noise. Canudas et al. [9] also proposed a model-based, adaptive friction compensation method for the model of [7] that was experimentally tested on a DC motor servomechanism. For the dynamic model of [7], Lischinsky et al. [19] developed a model-based friction compensation scheme for a six degree of freedom Schilling Titan II hydraulic robot. Panteley et al. [21] developed a global position tracking controller for an $n$-degree of freedom robot manipulator perturbed by friction forces (i.e., the friction compensation problem is viewed as a disturbance rejection problem). In [10], Canudas et al. proposed a passivity-based friction compensation scheme based on the friction model of [7] to achieve global asymptotic position tracking. In [25], Vedagarbha et al. constructed three observer/controller schemes that achieved different transient performance specifications assuming exact model knowledge. In addition, Vedagarbha et al. [25] proposed two adaptive observer-based control strategies to compensate for selected parametric uncertainty. In [23], Sivakumar et al. developed a robust, adaptive, variable structure controller to minimize the effects of friction described by the dynamic friction model of [7] in positioning applications. Jain et al. [17] proposed a robust adaptive controller to compensate for dynamic friction effects separated from the control input through drive compliance. The controller of [17] does not require any knowledge of the parameters associated with model [7] except for an upperbound on the static friction level to achieve setpoint regulation. In [26], Yazdizadeh et al. illustrated a Lyapunov-based design strategy for constructing a nonlinear estimator to compensate for the Coulomb friction coefficient to ensure asymptotic stability without placing any assumptions on the velocity.

In this paper, we extend the observer based control strategies of [25] to an n-link, direct drive, rigid link revolute robot. Specifically in Section II, we present the dynamic model of a robot manipulator system operating in the presence of nonlinear friction effects. In Section III, the various error signals necessary to facilitate the control development are defined. We develop a partial-state exact model knowledge, observer based control strategy to achieve global exponential link position tracking in Section IV. In Section V, we develop two adaptive, partialstate feedback controllers that actively compensate for selected parametric uncertainty in the system model and

\footnotetext{
${ }^{2}$ We note that there is a slight modification in the definition of $f(\dot{q})$ from that of [25] (i.e., $\beta_{2 i}$ is not squared in (4))
}

achieve global asymptotic position tracking. The first adaptive controller compensates for all model parameters except those associated with the nonlinear parameterizable Stribeck effect. The second adaptive controller addresses the normal variations associated with the Stribeck effect (i.e., all other system parameters are assumed to be known). In Section VI, we present a new adaptive control technique for compensating for the nonlinear parameterizable Stribeck effect. Specifically, an adaptive observer/controller scheme is developed which contains a feedforward approximation of the Stribeck effect. This feedforward approximation is used in a composite controller/observer strategy which forces the average square integral of the position tracking error to an arbitrarily small value. Experimental results are presented in Section VII.

\section{ROBOT MANIPULATOR MODEL AND PROPERTIES}

The dynamic model for a $n$-link, serially connected, direct drive, rigid-link revolute robot operating in the presence of nonlinear dynamic friction effects [7] can be expressed by the following

$$
\begin{aligned}
& M(q) \ddot{q}+V_{m}(q, \dot{q}) \dot{q}+G(q)+F_{d} \dot{q}+\chi(\dot{q}) z=\tau \\
& \dot{z}=\dot{q}-f(\dot{q}) z
\end{aligned}
$$

where the auxiliary functions $\chi(\dot{q}) \in \Re^{n \times n}$ and $f(\dot{q}) \in$ $\mathfrak{R}^{n \times n}$ are explicitly defined as $^{2}$

$$
\chi(\dot{q})=\theta_{0}-\theta_{1} f(\dot{q})
$$

$$
\begin{aligned}
& f(\dot{q})=\operatorname{diag}\left\{f_{i}\left(\dot{q}_{i}\right)\right\}=\operatorname{diag}\left\{\frac{\left|\dot{q}_{i}\right|}{\beta_{0 i}+\beta_{1 i} \exp \left(\frac{\dot{q}_{i}^{2}}{\beta_{2 i}}\right)}\right\} \\
& \text { for } i=1, \ldots, n,
\end{aligned}
$$

where $q(t), \dot{q}(t), \ddot{q}(t) \in \Re^{n \times n}$ denote the link position, velocity, and acceleration vectors, respectively, $M(q) \in$ $\Re^{n \times n}$ denotes the inertia matrix, $V_{m}(q, \dot{q}) \in \Re^{n \times n}$ denotes the centripetal-Coriolis matrix, $G(q) \in \mathfrak{R}^{n}$ denotes the gravity vector, $F_{d} \in \Re^{n \times n}$ denotes the positive definite diagonal matrix representing the viscous friction effects, $z(t) \in \mathfrak{R}^{n}$ denotes the unmeasurable internal friction state vector, $\theta_{0} \in \Re^{n \times n}$ and $\theta_{1} \in \Re^{n \times n}$ are positive definite, constant diagonal matrices that weight the friction effects, $f(\dot{q}) \in$ $\Re^{n \times n}$ denotes a diagonal matrix used to capture the Stribeck frictional effect $\left(\beta_{0 i}, \beta_{1 i}\right.$, and $\beta_{2 i}$ are positive, constant parameters), and $\tau(t) \in \mathfrak{R}^{n}$ represents the torque input control vector.

The robot dynamics of (1) are assumed to exhibit the following properties that will be exploited in the control development/stability analysis: 
Property 1. The inertia matrix $M(q)$ is symmetric, positive definite, and satisfies the following inequality

$$
m_{1}\|y\|^{2} \leq y^{T} M(q) y \leq m_{2}\|y\|^{2} \quad \forall y \in \Re^{n}
$$

where $m_{1}$ and $m_{2}$ are known positive, scalar constants, and $\|\cdot\|$ denotes the standard Euclidean norm.

Property 2. The inertia and the centripetal-Coriolis matrices exhibit the following property

$$
y^{T}\left(\frac{1}{2} \dot{M}(q)-V_{m}(q, \dot{q})\right) y=0 \quad \forall y \in \mathfrak{R}^{n}
$$

where $\dot{M}(q)$ denotes the time derivative of the inertia matrix.

Property 3. The robot dynamics of (1) are linearly parameterizable in the following manner

$$
Y(\ddot{q}, \dot{q}, q) \theta=M(q) \ddot{q}+V_{m}(q, \dot{q}) \dot{q}+G(q)+F_{d} \dot{q}
$$

where the regression matrix $Y(\ddot{q}, \dot{q}, q) \in \mathfrak{R}^{n \times p}$ contains known functions of the link acceleration, link velocity, and link position signals, and $\theta \in \mathfrak{R}^{p}$ contains the constant system parameters. It is assumed that if $q(t), \dot{q}(t), \ddot{q}(t)$ are bounded, then $Y(q, \dot{q}, \ddot{q}) \in L_{\infty}$.

Remark 1. The nonlinear state vector $z(t)$ [7] has been introduced in order to accurately take into account the stick-slip friction phenomenon occurring on a microscopic level between two contact surfaces. Conceptually, this model views the friction created between the two interacting surfaces as being caused by a large number of bristles in the narrow interstices of the rubbing surfaces. The state vector $z(t)$ used in the mechanical model of (1) and (2) represents the average deflection of thousands of such bristles. Clearly, the state $z(t)$ does not proffer itself for direct measurement.

Remark 2. We note that the structure of the function ${ }^{3}$ $f(\dot{q})$ ensures that $f(\dot{q}) \in L_{\infty}$ if $\dot{q}(t) \in L_{\infty}$. As illustrated in [7], the structure of $f(\dot{q})$ of (4) can be shown to guarantee that the internal friction state $z(t)$ is bounded. That is, a simple Lyapunov analysis applied to (2) yields

$$
\|z(t)\| \leq \delta_{0}
$$

where $\delta_{0}$ is some positive constant.

\footnotetext{
${ }^{3}$ We note that the definition of the function $f(\dot{q})$ of (4) utilizes a typical Stribeck capturing function as defined in Remark 3 of [25].
}

\section{PROBLEM FORMULATION}

In order to achieve the control objective of link position tracking for the dynamic model of (1) and (2) (under the assumption that link position and link velocity measurements are available), we define the link position tracking error $e(t) \in \mathfrak{R}^{n}$ as

$$
e=q_{d}-q
$$

where $q_{d}(t) \in \Re^{n}$ represents the desired link position trajectory. We assume that $q_{d}(t)$ and its corresponding first and second time derivative are bounded functions of time. In addition, we define the filtered tracking error $r(t) \in \mathfrak{R}^{n}$ as follows

$$
r=\dot{e}+\alpha e
$$

where $\alpha \in \mathfrak{R}^{n \times n}$ is a diagonal, positive definite, control gain matrix. To account for the inability to measure the internal friction state $z(t)$, we define the following observation error signal $\tilde{z}(t) \in \mathfrak{R}^{n}$ as follows

$$
\tilde{z}=z-\hat{z}
$$

where $\hat{z}(t) \in \mathfrak{R}^{n}$ represents the yet to be designed dynamic estimate for the unmeasurable friction state $z(t)$.

\section{EXACT MODEL KNOWLEDGE CONTROL DESIGN}

In this section, we design an exact model knowledge observer/controller to achieve link position tracking. That is, we assume that all of the system parameters of (1) and (2) are known exactly. Since we must provide for the observation of the internal friction state $z(t)$, we design an exact model knowledge observer that exploits the mechanical system dynamics of (1) in order to provide for convergence of the observation error (i.e., $\tilde{z}(t)$ will be driven to zero). The stability of the proposed observer/ controller is analyzed through Lyapunov based arguments.

\section{Controller design}

In this section, we design the torque control input $\tau(t)$ to promote link position tracking. To simplify the control development, we rewrite the mechanical dynamics of (1) in terms of the filtered tracking error $r(t)$ as follows

$$
\begin{aligned}
M(q) \dot{r}= & M(q)\left(\ddot{q}_{d}+\alpha \dot{e}\right)+V_{m}(q, \dot{q}) \dot{q}+G(q) \\
& +F_{d} \dot{q}+\chi(\dot{q}) z-\tau
\end{aligned}
$$

where (1) has been utilized. In order to prepare for the stability analysis, we add and subtract the term $V_{m}(q, \dot{q}) r$ to the right hand side of (12) to obtain the following expression 


$$
M(q) \dot{r}=-V_{m}(q, \dot{q}) r+W_{m}(q, \dot{q})+\chi(\dot{q}) z-\tau
$$

where the auxiliary signal $W_{m}(q, \dot{q}, t) \in \mathfrak{R}^{n}$ is explicitly defined as

$$
\begin{aligned}
W_{m}(q, \dot{q})= & M(q)\left(\ddot{q}_{d}+\alpha \dot{e}\right)+V_{m}(q, \dot{q})\left(\dot{q}_{d}+\alpha e\right) \\
& +G(q)+F_{d} \dot{q} .
\end{aligned}
$$

Based on the structure of the open-loop dynamics of (13), we design the control input vector $\tau(t)$ in the following manner

$$
\tau=W_{m}(q, \dot{q})+\chi(\dot{q}) \hat{z}+k_{s} r
$$

where $k_{s} \in \Re^{n \times n}$ is a diagonal, positive definite, control gain matrix. After substituting $\tau(t)$ of (15) into (13) and cancelling common terms, we obtain the closed loop filtered tracking error dynamics as follows

$$
M(q) \dot{r}=-V_{m}(q, \dot{q}) r-k_{s} r+[\chi(\dot{q}) \tilde{z}]
$$

where the definition of (11) has been utilized.

\section{Observer design}

Since the internal friction state $z(t)$ does not lend itself for practical measurement, we design an appropriate dynamic estimate $\hat{z}(t)$ for use in the control torque vector $\tau(t)$ of (15). Based on the structure of (2) and the ensuing stability analysis, we design the estimate $\hat{z}(t)$ as follows

$$
\hat{z}=p-\theta_{1}^{-1} M(q) \dot{q}
$$

where the dynamics for the internal variable $p(t) \in \Re^{n}$ are given by

$$
\begin{gathered}
\dot{p}=\theta_{1}^{-1}\left[-\theta_{0} \hat{z}+\dot{M}(q) \dot{q}-V_{m}(q, \dot{q}) \dot{q}-G(q)-F_{d} \dot{q}+\tau\right. \\
\left.+\theta_{1} \dot{q}+\chi(\dot{q}) r\right]
\end{gathered}
$$

After taking the time derivative of (17), pre-multiplying the result by the matrix $\theta_{1}$, substituting in the dynamic model of (1), and simplifying the resulting expression, we obtain the following non-implementable dynamics for $\hat{z}(t)$

$$
\begin{aligned}
\theta_{1} \dot{\hat{z}} & =-\theta_{0} \hat{z}+\theta_{1} \dot{q}+\chi(\dot{q}) r+\chi(\dot{q}) z \\
& =\theta_{0} \tilde{z}+\chi(\dot{q}) r+\theta_{1} \dot{q}-\theta_{1} f(\dot{q}) z
\end{aligned}
$$

where the definition of (3) and (11) has been utilized. After taking the time derivative of (11), pre-multiplying through by the matrix $\theta_{1}$, substituting in (2) and (19), and cancelling the common terms, we obtain the following expression for the closed-loop observation dynamics for $\tilde{z}(t)$

$$
\theta_{1} \dot{\tilde{z}}=-\theta_{0} \tilde{z}-\chi(\dot{q}) r
$$

Remark 3. We note that the term $\chi(\dot{q}) r$ has been injected into (18) to cancel the bracketed interconnection term in (16) during the subsequent stability analysis.

\section{Stability analysis}

In order to examine the stability of the proposed observer/controller, we define the following non-negative, scalar function as follows

$$
V_{0}=\frac{1}{2} r^{T} M(q) r+\frac{1}{2} \tilde{z}^{T} \theta_{1} \tilde{z}
$$

We note that $V_{0}(t)$ of (21) can be upper and lower bounded by the following inequalities

$$
\lambda_{1}\|\mathbf{x}\|^{2} \leq V_{0} \leq \lambda_{2}\|\mathbf{x}\|^{2}
$$

where the composite vector $\mathbf{x}(t) \in \mathfrak{R}^{2 n}$ is explicitly defined as

$$
\mathbf{x}=\left[\begin{array}{ll}
r^{T} & \tilde{z}^{T}
\end{array}\right]^{T},
$$

$\lambda_{1}$ and $\lambda_{2}$ are positive, scalar, constants given by

$$
\begin{aligned}
& \lambda_{1}=\frac{1}{2} \min \left\{m_{1}, \lambda_{\min }\left\{\theta_{1}\right\}\right\} \\
& \lambda_{2}=\frac{1}{2} \max \left\{m_{2}, \lambda_{\max }\left\{\theta_{1}\right\}\right\}
\end{aligned}
$$

where $\lambda_{\min }\{\cdot\}$ and $\lambda_{\max }\{\cdot\}$ denote the minimum/maximum eigenvalue of a matrix, respectively. After taking the time derivative of $V_{0}(t)$, substituting in (16) and (20), utilizing (6), and cancelling the resulting common terms, we obtain the following expression for $\dot{V}_{0}(t)$

$$
\dot{V}_{0}=-r^{T} k_{s} r-\tilde{z}^{T} \theta_{0} \tilde{z} \leq-\lambda_{3}\|\mathbf{x}\|^{2}
$$

where the positive, scalar constant $\lambda_{3}$ is given by

$$
\lambda_{3}=\min \left\{\lambda_{\min }\left\{k_{s}\right\}, \lambda_{\min }\left\{\theta_{0}\right\}\right\} .
$$

After utilizing the inequalities of (22), we can rewrite $\dot{V}_{0}(t)$ of (25) in the following manner

$$
\dot{V}_{0} \leq-\frac{\lambda_{3}}{\lambda_{2}} V_{0}
$$

After solving the differential inequality of (27) and 
utilizing the inequality given by (22), we obtain the following expression for the composite vector $\mathbf{x}(t)$

$$
\|x(t)\| \leq \sqrt{\frac{\lambda_{2}}{\lambda_{1}}} \exp \left(-\frac{\lambda_{3}}{2 \lambda_{2}}\right)\|x(0)\| .
$$

Clearly from (28), we can see that $\mathbf{x}(t)$ goes to zero exponentially fast; hence, from the definition of (23), we know that the filtered position tracking error variable $r(t)$ and the observation error $\tilde{z}(t)$ also go to zero exponentially fast. Since the filtered tracking error variable $r(t)$ is related to $e(t)$ through an exponentially stable transfer function, we can then show that the link position tracking error $e(t)$ converges to zero exponentially [24]. Standard signal chasing arguments can be used to show that all signals in the controller/observer and the mechanical system remain bounded during closed-loop operation.

\section{ADAPTIVE CONTROL DESIGN}

In this section, we present the two adaptive observer/ controller schemes of [25] to compensate for parametric uncertainty in selected parameters of the mechanical system of (1).

\section{Case 1 - known stribeck function}

In this subsection, we propose an adaptive partialstate feedback control for the mechanical system of (1) and (2) that compensates for all the parameters except those appearing in $f(\dot{q})$. That is, we assume that $f(\dot{q})$ is available for use in the design of the observer/control algorithm. After rewriting the mechanical system of (1) in terms of the filtered tracking error variable $r(t)$ as done similarly in (13), we obtain

$$
M(q) \dot{r}=-V_{m}(q, \dot{q}) r+Y_{m} \theta_{m}+\theta_{0} z-\theta_{1} f(\dot{q}) z-\tau
$$

where the definition of (3) has been used, and (7) has been utilized to define the regression matrix/parameter vector formulation as

$$
\begin{aligned}
Y_{m} \theta_{m}= & M(q)\left(\ddot{q}_{d}+\alpha \dot{e}\right)+V_{m}(q, \dot{q})\left(\dot{q}_{d}+\alpha e\right) \\
& +G(q)+F_{d} \dot{q}
\end{aligned}
$$

where $Y_{m}\left(\ddot{q}_{d}, q_{d}, q_{d}, \dot{q}, q, t\right) \in \mathfrak{R}^{n \times p}$ denotes a known regression matrix, and $\theta_{m} \in \mathfrak{R}^{p}$ represents a vector of unknown, constant, system parameters. Based on the open-loop structure of (29) and the ensuing Lyapunov stability analysis, we design the torque input vector as

$$
\tau=k_{s} r+Y_{m} \hat{\theta}_{m}+\hat{\theta}_{0} \hat{z}-\hat{\theta}_{1} f(\dot{q}) \hat{z}+\hat{\theta}_{0} \zeta_{0}-\hat{\theta}_{1} f(\dot{q}) \zeta_{1}
$$

where $k_{s} \in \mathfrak{R}^{n \times n}$ is defined as a diagonal, positive definite, control gain matrix, $\hat{\theta}_{m}(t) \in \mathfrak{R}^{p}, \widehat{\theta}_{0}(t) \in \mathfrak{R}^{n \times n}, \hat{\theta}_{1}(t) \in$ $\Re^{n \times n}$ represent the dynamic parameter estimates for $\theta_{m}, \theta_{0}$, $\theta_{1}$, respectively, which are calculated on-line from the following update laws

$$
\begin{aligned}
& \dot{\hat{\theta}}_{m}=\Gamma_{m} Y_{m}^{T} r \quad \dot{\hat{\theta}}_{0 i}=\gamma_{0 i}\left(\hat{z}_{i}+\zeta_{0 i}\right) r_{i} \\
& \dot{\hat{\theta}}_{1 i}=-\gamma_{1 i} f_{i}\left(\dot{q}_{i}\right)\left(\hat{z}_{i}+\zeta_{1 i}\right) r_{i} \text { for } i=1, \ldots, n,
\end{aligned}
$$

where $\Gamma_{m} \in \mathfrak{R}^{p \times p}$ denotes a diagonal, positive definite, adaptation gain matrix, $\gamma_{0 i}, \gamma_{1 i}$ denote positive, constant, scalar adaptation gains, and $\zeta_{0}(t), \zeta_{1}(t) \in \mathfrak{R}^{n}$ are nonlinear, auxiliary filter signals with the following dynamics

$$
\dot{\zeta}_{0}=-f(\dot{q}) \zeta_{0}+r \quad \dot{\zeta}_{1}=-f(\dot{q}) \zeta_{1}-f(\dot{q}) r
$$

After substituting $\tau(t)$ of (31) into (29) and simplifying the result, we obtain the following expression for the closed-loop filter tracking error dynamics as given by

$$
\begin{aligned}
M(q) \dot{r}= & -k_{s} r-V_{m}(q, \dot{q}) r+Y_{m} \tilde{\theta}_{m}+\tilde{\theta}_{0}\left(\hat{z}+\zeta_{0}\right) \\
& -\tilde{\theta}_{1} f(\dot{q})\left(\hat{z}+\zeta_{1}\right)+\theta_{0}\left(\tilde{z}-\zeta_{0}\right) \\
& -\theta_{1} f(\dot{q})\left(\tilde{z}-\zeta_{1}\right)
\end{aligned}
$$

where $\tilde{\theta}_{m}(t)=\theta_{m}-\hat{\theta}_{m}(t) \in \mathfrak{R}^{p}, \tilde{\theta}_{0}(t)=\theta_{0}-\hat{\theta}_{0}(t) \in \mathfrak{R}^{n \times n}$, and $\tilde{\theta}_{1}(t)=\theta_{1}-\tilde{\theta}_{1}(t) \in \Re^{n \times n}$ denote the corresponding parameter estimation error. We note that $\hat{\theta}_{0 i}(t), \hat{\theta}_{1 i}(t)$ denote parameter estimates for $i^{\text {th }}$ main diagonal elements of $\theta_{0}, \theta_{1}$, respectively; hence, $\tilde{\theta}_{0}(t)$ and $\tilde{\theta}_{1}(t)$ are only updated on the main diagonal elements. Based on the structure of (2), we design the dynamics for the observer $\hat{z}(t)$ as follows

$$
\dot{\hat{z}}=\dot{q}-f(\dot{q}) \hat{z}
$$

After taking the time derivative of (11), substituting in (2) and (35), and cancelling common terms, we obtain the following

$$
\dot{\tilde{z}}=-f(\dot{q}) \hat{z}
$$

Remark 4. We note that (35) represents a rather simplistic method for designing the observer $\hat{z}(t)$ motivated from rewriting the dynamic equation governing the unmeasurable state (2) in terms of the estimated state. We note that we are forced to construct this simple observer due to the fact that many of the system parameters are not known.

In order to examine the stability of the proposed adaptive observer/controller of (31), (35), (32), and (33), we define the following non-negative, scalar function $V_{1}(t)$ as follows 


$$
\begin{aligned}
V_{1}= & \frac{1}{2} r^{T} M(q) r+\frac{1}{2} \tilde{z}^{T} \tilde{z}+\frac{1}{2}\left(\tilde{z}-\zeta_{0}\right)^{T} \theta_{0}\left(\tilde{z}-\zeta_{0}\right) \\
& +\frac{1}{2}\left(\tilde{z}-\zeta_{1}\right)^{T} \theta_{1}\left(\tilde{z}-\zeta_{1}\right)+\frac{1}{2} \tilde{\theta}_{m}^{T} \Gamma_{m}^{-1} \tilde{\theta}_{m} \\
& +\frac{1}{2} \sum_{i=1}^{n} \frac{1}{\gamma_{0 i}} \tilde{\theta}_{0 i}^{2}+\frac{1}{2} \sum_{i=1}^{n} \frac{1}{\gamma_{1 i}} \tilde{\theta}_{1 i}^{2}
\end{aligned}
$$

After taking the time derivative of (37), substituting in (34), (35), and (33), and simplifying the resulting expression, we obtain the following expression for $\dot{V}_{1}(t)$

$$
\begin{aligned}
\dot{V}_{1}= & -r^{T} k_{s} r-\tilde{z}^{T} f(\dot{q}) \tilde{z}+r^{T} Y_{m} \tilde{\theta}_{m}-\tilde{\theta}_{m}^{T} \Gamma_{m}^{-1} \dot{\hat{\theta}}_{m} \\
& +\left(\left[r^{T} \tilde{\theta}_{0}\left(\hat{z}+\zeta_{0}\right)\right]-\sum_{i=1}^{n} \tilde{\theta}_{0 i} \frac{1}{\gamma_{0 i}} \dot{\hat{\theta}}_{0 i}\right) \\
& +\left(-\left[r^{T} \tilde{\theta}_{1} f(\dot{q})\left(\hat{z}+\zeta_{1}\right)\right]-\sum_{i=1}^{n} \tilde{\theta}_{1 i} \frac{1}{\gamma_{1 i}} \dot{\hat{\theta}}_{1 i}\right) \\
& -\left(\tilde{z}-\zeta_{0}\right)^{T} \theta_{0} f(\dot{q})\left(\tilde{z}-\zeta_{0}\right)-\left(\tilde{z}-\zeta_{1}\right)^{T} \theta_{1} f(\dot{q})\left(\tilde{z}-\zeta_{1}\right)
\end{aligned}
$$

where (6) has been utilized. Since $\tilde{\theta}_{0}(t)$ and $\tilde{\theta}_{1}(t)$ are main diagonal matrices, the bracketed terms of (38) can be written in the following manner

$$
\begin{aligned}
& r^{T} \tilde{\theta}_{0}\left(\hat{z}+\zeta_{0}\right)=\sum_{i=1}^{n} \tilde{\theta}_{0 i}\left(\hat{z}_{i}+\zeta_{0 i}\right) r_{i} \\
& r^{T} \tilde{\theta}_{1} f(\dot{q})\left(\hat{z}+\zeta_{1}\right)=\sum_{i=1}^{n} \tilde{\theta}_{1 i} f_{i}\left(\dot{q}_{i}\right)\left(\hat{z}_{i}+\zeta_{1 i}\right) r_{i}
\end{aligned}
$$

where $f_{i}\left(\dot{q}_{i}\right)$ denotes the $i_{t h}$ diagonal element of $f(\dot{q})$. After utilizing (39) and substituting in the parameter update laws of (32), we obtain

$$
\begin{aligned}
\dot{V}_{1}= & -r^{T} k_{s} r-\tilde{z}^{T} f(\dot{q}) \tilde{z}-\left(\tilde{z}-\zeta_{0}\right)^{T} \theta_{0} f(\dot{q})\left(\tilde{z}-\zeta_{0}\right) \\
& -\left(\tilde{z}-\zeta_{1}\right)^{T} \theta_{1} f(\dot{q})\left(\tilde{z}-\zeta_{1}\right),
\end{aligned}
$$

which can be upperbounded in the following manner

$$
\dot{V}_{1} \leq-r^{T} k_{s} r
$$

From the fact that $V_{1}(t)$ is a non-negative function, and $\dot{V}_{1}(t)$ is non-positive, we can state that $V_{1}(t)$ is either decreasing or constant; hence, $V_{1}(t) \in L_{\infty}$. Therefore, all signals contained in $V_{1}(t)$ of (37) remain bounded (i.e., $r(t)$, $\left.\tilde{z}(t), \tilde{\theta}_{m}(t), \tilde{\theta}_{0}, \tilde{\theta}_{1}, \zeta_{0}(t), \zeta_{1}(t) \in L_{\infty}\right)$. Since $\tilde{z}(t), z(t) \in L_{\infty}$ (See Remark 2), we can show that the dynamic observer $\hat{z}(t) \in L_{\infty}$. Hence, from (34), we can prove that $\dot{r}(t) \in L_{\infty}$. From the fact that $V_{1}(t)$ is decreasing or constant and the structure of (41), we can easily illustrate that $r(t) \in L_{2}$. Hence, direct application of Barbalat's Lemma [22] and standard linear control arguments yield

$$
\lim _{t \rightarrow \infty} r(t)=0 \Rightarrow \lim _{t \rightarrow \infty} e(t), \dot{e}(t)=0 .
$$

Standard signal chasing arguments can be used to show that all signals in the controller/observer and the mechanical system remain bounded during closed-loop operation.

\section{Case 2 - unknown normal force variation parameter}

We now develop an adaptive observer/controller scheme that addresses the parametric uncertainty problem associated with normal force variation in the Stribeck effect. That is, we assume that the matrix $f(\dot{q})$ can be expressed as follows

$$
f(\dot{q})=k f_{k}(\dot{q})
$$

where $k \in \mathfrak{R}^{n \times n}$ denotes a diagonal matrix of unknown positive constants, and $f_{k}(\dot{q}) \in \mathfrak{R}^{n \times n}$ represents a diagonal matrix composed of known functions (Note that all other system parameters of (1) are assumed to be known). After rewriting (1) in terms of the filtered tracking error variable as done similarly in (13), we obtain the following expression

$$
M(q) \dot{r}=-V_{m}(q, \dot{q}) r+W_{m}(q, \dot{q})+\theta_{0} z-\theta_{1} k f_{k}(\dot{q}) z-\tau
$$

where $W_{m}(q, \dot{q})$ was previously defined in (14), and (43) has been utilized. Based on the open-loop structure of (44), we design the torque input $\tau(t)$ as follows

$$
\tau=k_{s} r+W_{m}(q, \dot{q})+\theta_{0} \hat{z}-\theta_{1} \hat{k} f_{k}(\dot{q}) \hat{z}-\theta_{1} \hat{k} f_{k}(\dot{q}) \zeta_{k}
$$

where $k_{s} \in \mathfrak{R}^{n \times n}$ is a diagonal matrix of positive, constant control gains, $\hat{k}(t) \in \mathfrak{R}^{n \times n}$ denotes the dynamic estimate for the diagonal matrix $k$ which is calculated using the following update law

$$
\dot{\hat{k}}_{i}=-\gamma_{k i} \theta_{1 i} f_{k i}\left(\dot{q}_{i}\right)\left(\hat{z}_{i}+\zeta_{k i}\right) r_{i} \text { for } i=1, \ldots, n,
$$

where $\gamma_{k i}$ is a positive, scalar adaptation gain, and $\zeta_{k}(t) \in \mathfrak{R}^{n}$ is an auxiliary filter state vector calculated on-line from the following dynamic expression

$$
\zeta_{k}=\theta_{1}^{-1}\left[-f_{k}(\dot{q}) r-\theta_{0} r-\theta_{0} \zeta_{k}\right]
$$

We note that $\hat{k}_{i}(t)$ denotes the parameter estimate for $i^{\text {th }}$ main diagonal element of $k$; hence, $\hat{k}_{i}(t)$ is only updated on the main diagonal elements.

After substituting $\tau(t)$ of (45) into (44) and cancelling common terms, we obtain the following closed-loop expression for the filtered tracking error dynamics

$$
M(q) \dot{r}=-k_{s} r-V_{m}(q, \dot{q}) r-\theta_{1} \tilde{k} f_{k}(\dot{q})\left(\hat{z}+\zeta_{k}\right)
$$




$$
-\theta_{1} k f_{k}(\dot{q})\left(\tilde{z}-\zeta_{k}\right)+\left[\theta_{0} \tilde{z}\right]
$$

where $\tilde{k}(t)=k-\hat{k} \in \mathfrak{R}^{n \times n}$ is a diagonal matrix denoting the corresponding parameter estimation error for the parameter matrix $k$. Based on the ensuing Lyapunov stability analysis, we propose the following observer for the internal friction state

$$
\hat{z}=p-\theta_{1}^{-1} M(q) \dot{q}
$$

where the dynamics for the internal variable $p(\mathrm{t}) \in \mathfrak{R}^{n}$ are given by the following expression

$$
\begin{aligned}
\dot{p}= & \theta_{1}^{-1}\left[-\theta_{0} \hat{z}+\dot{M}(q) \dot{q}+\left(-V_{m}(q, \dot{q}) \dot{q}-G(q)-F_{d} \dot{q}\right.\right. \\
& \left.+\tau)+\theta_{0} r+\theta_{1} \dot{q}\right]
\end{aligned}
$$

After taking the time derivative of (49), substituting in (1) and (50), pre-multiplying through by the matrix $\theta_{1}$, and cancelling common terms, we obtain the following expression for the non-implementable dynamics for $\hat{z}(t)$

$$
\theta_{1} \dot{\hat{z}}=\theta_{0} \tilde{z}+\theta_{0} r+\theta_{1} \dot{q}-\theta_{1} k f_{k}(\dot{q}) z
$$

where the definition of (11) has been utilized. After taking the time derivative of the observation error of (11), premultiplying by the matrix $\theta_{1}$, substituting in the expressions of (2) and (51), and cancelling common terms, we obtain the following expression for the closed-loop observation error dynamics for $\tilde{z}(t)$ as given by

$$
\theta_{1} \dot{\hat{z}}=-\theta_{0} \tilde{z}-\left[\theta_{0} r\right]
$$

Remark 5. We note that bracketed term of (52) has been injected in the observer construction in order to cancel the bracketed term of (48) during the ensuing stability analysis.

To analyze the stability of the proposed observer/ controller scheme, we define the following non-negative, scalar function

$$
\begin{aligned}
V_{2}= & \frac{1}{2} r^{T} M(q) r+\frac{1}{2} \tilde{z}^{T} \theta_{1} \tilde{z}+\frac{1}{2}\left(\tilde{z}-\zeta_{k}\right)^{T} \theta_{1} k \theta_{1}\left(\tilde{z}-\zeta_{k}\right) \\
& +\frac{1}{2} \sum_{i=1}^{n} \frac{1}{\gamma_{k i}} \tilde{k}_{i}^{2} .
\end{aligned}
$$

After taking the time derivative of (53), substituting in the dynamics of (48), (52), and (47), and simplifying the resulting expression, we obtain the following expression for $\dot{V}_{2}(t)$

$$
\dot{V}_{2}=-r^{T} k_{s} r-\tilde{z}^{T} \theta_{0} \tilde{z}-\left[r^{T} \theta_{1} \tilde{k} f_{k}(\dot{q})\left(\hat{z}+\zeta_{k}\right)\right]
$$

$$
-\left(\tilde{z}-\zeta_{k}\right)^{T} \theta_{1} k \theta_{0}\left(\tilde{z}-\zeta_{k}\right)-\sum_{i=1}^{n} \tilde{k}_{i} \frac{1}{\gamma_{k i}} \dot{\hat{k}}_{i} .
$$

We note that the bracketed term of (54) can be rewritten as follows

$$
r^{T} \theta_{1} \tilde{k} f_{k}(\dot{q})\left(\hat{z}+\zeta_{k}\right)=\sum_{i=1}^{n} \theta_{1 i} \tilde{k}_{i} f_{k i}\left(\dot{q}_{i}\right)\left(\hat{z}_{i}+\zeta_{k i}\right) r_{i}
$$

where we have utilized the fact that the matrices $\theta_{1}, \tilde{k}(t)$, and $f_{k}(\dot{q})$ are diagonal. After substituting (55) and (46) into (54), we obtain the following

$$
\dot{V}_{2}=-r^{T} k_{s} r-\tilde{z}^{T} \theta_{0} \tilde{z}-\left(\tilde{z}-\zeta_{k}\right)^{T} \theta_{1} k \theta_{0}\left(\tilde{z}-\zeta_{k}\right)
$$

which can be upperbounded in the following manner

$$
\dot{V}_{2} \leq-r^{T} k_{s} r-\tilde{z}^{T} \theta_{0} \tilde{z}
$$

where we have utilized the fact that $\theta_{1} k \theta_{0}$ is a positive definite matrix.

From the fact that $k_{s}$ and $\theta_{0}$ are positive definite, diagonal matrices, we can see from (57) that $\dot{V}(t)$ is negative semi-definite. Therefore, we can state that the nonnegative function $V(t)$ is decreasing or constant; hence, $V(t)$ is bounded (i.e., $r(t), \tilde{z}(t), \tilde{k}(t), \zeta_{k}(t) \in L_{\infty}$ ). From Remark 2 and the fact $\tilde{z}(t) \in L_{\infty}$, we know that the observer signal $\hat{z}(t) \in L_{\infty}$. Since $r(t) \in L_{\infty}$, we can state that $q(t), \dot{q}(t) \in L_{\infty}$, since $q_{d}(t), \dot{q}_{d}(t)$ are assumed to be bounded. From (48), we can state the $\dot{r}(t) \in L_{\infty}$. From the structure of (57), we can easily show that $r(t) \in L_{2}$. Hence, direct application of Barbalat's Lemma [22] and standard linear control arguments yield

$$
\lim _{t \rightarrow \infty} r(t)=0 \Rightarrow \lim _{t \rightarrow \infty} e(t), \dot{e}(t)=0 .
$$

Standard signal chasing arguments can be used to show that all signals in the controller/observer and the mechanical system remain bounded during closed-loop operation.

\section{ADAPTIVE COMPENSATION FOR THE STRIBECK EFFECT}

In this section, we develop an adaptive control strategy which compensates for parametric uncertainty associated with the nonlinearly parameterizable Stribeck effects. For simplicity, we only examine the one degree of freedom problem. Specifically, the mechanical system dynamics are now expressed in the following manner

$$
\begin{aligned}
& M \ddot{q}+B \dot{q}+T_{L}(q, \dot{q})+\chi(\dot{q}) z=u, \\
& \dot{z}=\dot{q}-f(\dot{q}) z,
\end{aligned}
$$

where the auxiliary scalar functions $\chi(\dot{q})$ and $f(\dot{q})$ are defined as follows 


$$
\begin{aligned}
& \chi(\dot{q})=\theta_{0}-\theta_{1} f(\dot{q}) \\
& f(\dot{q})=\frac{|\dot{q}|}{\beta_{0}+\beta_{1} \exp \left(-\frac{\dot{q}^{2}}{\beta_{2}}\right)},
\end{aligned}
$$

$q(t), \dot{q}(t), \ddot{q}(t)$ represent scalar position, velocity, and acceleration, respectively, $z(t)$ denotes the unmeasurable internal friction state, $M$ denotes the constant mechanical inertia of the system, $B$ denotes the constant viscous friction coefficient, $T_{L}(q, \dot{q})$ denotes a scalar nonlinear load function dependent on the position and velocity, $\theta_{i}, \beta_{i} \in \mathfrak{R}^{1}$ are positive constant parameters, and $u(t)$ is the control torque input. With regard to the above model, we will assume that all quantities are exactly known except for the constants $\beta_{0}, \beta_{1}$, and $\beta_{2}$.

Based on the structure of (62), we propose the following linear parameterizable function $\bar{f}(\dot{q})$ to approximate the function $f(\dot{q})$ as shown below

$$
\bar{f}(\dot{q}) \equiv \sigma_{0}|\dot{q}|+\sum_{k=1}^{m} \sigma_{k} \bar{m}_{k}(\dot{q})
$$

where the function $\bar{m}_{k}(\dot{q}) \in \Re^{1}$ is given by

$$
\bar{m}_{k}(\dot{q})=|\dot{q}| \tanh \left(\frac{\dot{q}^{2}}{k}\right)
$$

where $\sigma_{0}$ and $\sigma_{k^{\prime} s}$ denote unknown positive, constant scalar weighting constants, and $m$ represents the number of functions used to approximate $f(\dot{q})$. After rewriting the mechanical system of (1) in terms of the filtered tracking error $r(t)$, we obtain the following expression

$$
\begin{aligned}
M \dot{r}= & M\left(\ddot{q}_{d}+\alpha \dot{e}\right)+B \dot{q}+T_{L}(q, \dot{q})+\theta_{0} z-\theta_{1} f(\dot{q}) z \\
& +\left(\theta_{1} \bar{f}(\dot{q}) z-\theta_{1} \bar{f}(\dot{q}) z\right)-u
\end{aligned}
$$

where the definition of (61) has been utilized, and the term $\theta_{1} \bar{f}(\dot{q}) z$ has been added and subtracted to the right hand side of (65). After simplifying the parenthetical expression of (65), we obtain the following

$$
\begin{aligned}
M \dot{r}= & M\left(\ddot{q}_{d}+\alpha \dot{e}\right)+B \dot{q}+T_{L}(q, \dot{q})+\theta_{0} z-\theta_{1} \bar{f}(\dot{q}) z \\
& -\theta_{1} \tilde{f}(\dot{q}) z-u
\end{aligned}
$$

where $\tilde{f}(\dot{q})=f(\dot{q})-\bar{f}(\dot{q})$ represents the mismatch error

\footnotetext{
${ }^{4}$ The projection algorithm given by (70) ensures that if the adaptive estimates are initialized between the known upper and lower bounds for the unknown parameters, then the adaptive estimates will remain between the known upper and lower bounds for all time.
}

associated with the function $f(\dot{q})$.

Remark 6. The structure of (63) and (64) was motivated from an experimentally obtained profile of (62). We note the expressions of (63) and (64) represent only one possible approximating function for $f(\dot{q})$ and that the approximating method of (63) and (64) can be easily extended to an n-link, serially connected, rigid link, revolute robot. From the structure of (62) and (63), we note that the approximation error $\tilde{f}(\dot{q})$ can be upperbounded in the following manner

$$
\|\tilde{f}(\dot{q})\| \leq \rho\|\dot{q}\|
$$

where $\rho$ is some positive constant. It should be noted that the magnitude of $\rho$ will be smaller if we select larger values for $m$ in the approximating function given in (63).

Based on the structure of (66), we design the torque input $u(t)$ as follows

$$
\begin{aligned}
u= & k_{s} r+M\left(\ddot{q}_{d}+a \dot{e}\right)+B \dot{q}+T_{L}(q, \dot{q})+\theta_{0} \hat{z}-\theta_{1} \hat{f}(\dot{q}) \hat{z} \\
& -\theta_{1} \hat{\sigma}_{0}|\dot{q}| \zeta_{0}-\theta_{1} \sum_{k=1}^{m} \hat{\sigma}_{k} \bar{m}_{k}(\dot{q}) \zeta_{k}+k_{n}\left(\theta_{1} \dot{q}\right)^{2} r(68)
\end{aligned}
$$

where $k_{s}, k_{n}$ denotes positive, scalar control gains, and $\hat{f}(\dot{q})$ is explicitly defined as follows

$$
\widehat{f}(\dot{q})=\hat{\sigma}_{0}|\dot{q}|+\sum_{k=1}^{m} \hat{\sigma}_{k} \bar{m}_{k}(\dot{q})
$$

$\hat{\sigma}_{0}(t), \hat{\sigma}_{k}(t)$ denote weighting coefficient estimates that are calculated using the following projection based update law

$\dot{\hat{\sigma}}_{0}= \begin{cases}0 & \text { if }\left\{\begin{array}{l}\hat{\sigma}_{0}=\bar{\sigma}_{0} \text { and }-\gamma_{0} \theta_{1}|\dot{q}|\left(\hat{z}+\zeta_{0}\right) r>0 \\ \hat{\sigma}_{0}=\underline{\sigma}_{0} \text { and }-\gamma_{0} \theta_{1}|\dot{q}|\left(\hat{z}+\zeta_{0}\right) r<0\end{array}\right. \\ -\gamma_{0} \theta_{1}|\dot{q}|\left(\hat{z}+\zeta_{0}\right) r & \text { otherwise }\end{cases}$ $\dot{\hat{\sigma}}_{k}= \begin{cases}0 & \text { if }\left\{\begin{array}{l}\hat{\sigma}_{k}=\bar{\sigma}_{k} \text { and }-\gamma_{k} \theta_{1} \bar{m}_{k}(\dot{q})\left(\hat{z}+\zeta_{k}\right) r>0 \\ \hat{\sigma}_{k}=\underline{\sigma}_{k} \text { and }-\gamma_{k} \theta_{1} \bar{m}_{k}(\dot{q})\left(\hat{z}+\zeta_{k}\right) r<0\end{array}\right. \\ -\gamma_{k} \theta_{1} \bar{m}_{k}(\dot{q})\left(\hat{z}+\zeta_{k}\right) r & \text { otherwise }\end{cases}$

$\gamma_{0}, \gamma_{k^{\prime} s}$ are positive, constant adaptation gains, $\bar{\sigma}_{0}, \underline{\sigma}_{0}$ and $\bar{\sigma}_{k}, \underline{\sigma}_{k}$ represent the known upper and lower bounds ${ }^{4}$ for $\sigma_{0}$ and $\sigma_{k}$, respectively, $\zeta_{0}(t), \zeta_{k}(t)$ denote nonlinear, auxiliary filter variables that have the following dynamics 


$$
\begin{aligned}
& \dot{\zeta}_{0}=-\theta_{1}|\dot{q}| r-\frac{\theta_{0}}{\theta_{1}} r-\frac{\theta_{0}}{\theta_{1}} \zeta_{0} \\
& \dot{\zeta}_{k}=-\theta_{1} \bar{m}_{k}(\dot{q}) r-\frac{\theta_{0}}{\theta_{1}} r-\frac{\theta_{0}}{\theta_{1}} \zeta_{k},
\end{aligned}
$$

$k_{n}$ denotes a positive, constant, nonlinear damping gain, and $\hat{z}(t)$ represents the yet to be designed estimate for internal friction. After substituting in the control torque of (68) into the open-loop dynamics of (66) and simplifying the resulting expression, we obtain

$$
\begin{aligned}
M \dot{r}= & -k_{s} r+\theta_{0} \tilde{z}-\tilde{\sigma}_{0} \theta_{1}|\dot{q}|\left(\hat{z}+\zeta_{0}\right)-\theta_{1} \sigma_{0}|\dot{q}|\left(\tilde{z}-\zeta_{0}\right) \\
& -\theta_{1} \sum_{k=1}^{m} \tilde{\sigma}_{k} \bar{m}_{k}(\dot{q})\left(\hat{z}+\zeta_{k}\right)-\theta_{1} \sum_{k=1}^{m} \sigma_{k} \bar{m}_{k}(\dot{q})\left(\tilde{z}-\zeta_{k}\right) \\
& +\left(-\theta_{1} \tilde{f}(\dot{q}) z-k_{n}\left(\theta_{1} \dot{q}\right)^{2} r\right)
\end{aligned}
$$

where $\tilde{\sigma}_{0}(t)=\sigma_{0}-\hat{\sigma}_{0}$ and $\tilde{\sigma}_{k}=\sigma_{k}-\hat{\sigma}_{k}$ represent the parameter estimation error and the definitions of (63) and (69 have been substituted into (72).

For the scalar system of (59) and (60), we construct the following nonlinear observer for $\hat{z}(t)$ [25]

$$
\hat{z}=p-\frac{M}{\theta_{1}} \dot{q}
$$

where the internal scalar signal $p(t)$ has the following dynamics

$$
\dot{p}=\frac{1}{\theta_{1}}\left[-\theta_{0} \hat{z}-B \dot{q}-T_{L}(q, \dot{q})+u+\theta_{0} r+\theta_{1} \dot{q}\right]
$$

After taking the time derivative of (73), pre-multiplying through by the system parameter $\theta_{1}$, substituting in (59) and (74), and simplifying the resulting expression, we obtain the following non-implementable dynamics for the observer $z(t)$

$$
\theta_{1} \dot{\hat{z}}=\theta_{0} \tilde{z}+\theta_{0} r+\theta_{1} \dot{q}-\theta_{1} f(\dot{q}) z
$$

where we have utilized the definition of (61). After taking the time derivative of $\tilde{z}(t)$, pre-multiplying the result by the parameter $\theta_{1}$, substituting in the expressions of (60) and (75), and cancelling common terms, the closed-loop observation error dynamics for $\tilde{z}(t)$ is given by the following expression

$$
\theta_{1} \dot{\tilde{z}}=-\theta_{0} \tilde{z}-\theta_{0} r
$$

\footnotetext{
${ }^{5}$ The reader is referred to [6] for the explicit details which illustrate how the update law of (70) leads to the upper bound for $\dot{V}_{3}(t)$ given by $(80)$.
}

In order to analyze the overall stability of the system, we define the following non-negative, scalar function $V_{3}(t)$ as follows

$$
\begin{aligned}
V_{3}= & \frac{1}{2} M r^{2}+\frac{1}{2} \theta_{1} \tilde{z}^{2}+\frac{1}{2} \frac{1}{\gamma_{0}} \tilde{\sigma}_{0}^{2}+\frac{1}{2} \sum_{k=1}^{m} \frac{1}{\gamma_{k}} \tilde{\sigma}_{k}^{2} \\
& +\frac{1}{2} \sigma_{0}\left(\tilde{z}-\zeta_{0}\right)^{2}+\frac{1}{2} \sum_{k=1}^{m} \sigma_{k}\left(\tilde{z}-\zeta_{k}\right)^{2}
\end{aligned}
$$

We note that $V_{3}(t)$ of (77) can be upper and lower bounded in the following manner

$$
\begin{aligned}
\varepsilon_{1}\|\mathbf{y}\|^{2} & +\frac{1}{2} \frac{1}{\gamma_{0}} \tilde{\sigma}_{0}^{2}+\frac{1}{2} \sum_{k=1}^{m} \frac{1}{\gamma_{k}} \tilde{\sigma}_{k}^{2} \leq V_{3}(t) \leq \varepsilon_{2}\|\mathbf{y}\|^{2} \\
& +\frac{1}{2} \frac{1}{\gamma_{0}} \tilde{\sigma}_{0}^{2}+\frac{1}{2} \sum_{k=1}^{m} \frac{1}{\gamma_{k}} \tilde{\sigma}_{k}^{2}
\end{aligned}
$$

where the composite state vector $\mathbf{y}(t) \in \mathfrak{R}^{m+3}$ is explicitly defined as follows

$$
\mathbf{y}=\left[r, \tilde{z},\left(\tilde{z}-\zeta_{0}\right),\left(\tilde{z}-\zeta_{1}\right), \ldots,\left(\tilde{z}-\zeta_{m}\right)\right]^{T}
$$

and $\varepsilon_{1}, \varepsilon_{2}$ are positive scalar constants defined as follows

$$
\begin{aligned}
& \varepsilon_{1}=\frac{1}{2} \min \left\{M, \theta_{1}, \sigma_{0}, \sigma_{1}, \ldots, \sigma_{m}\right\} \\
& \varepsilon_{2}=\frac{1}{2} \max \left\{M, \theta_{1}, \sigma_{0}, \sigma_{1}, \ldots, \sigma_{m}\right\}
\end{aligned}
$$

After taking the time derivative of (77), substituting in the closed-loop dynamics of (72) and (76), the parameter update laws of (70), and the auxiliary filter dynamics of (71), and cancelling common terms, we obtain the following upper bound ${ }^{5}$ for $\dot{V}_{3}(t)$

$$
\begin{aligned}
\dot{V}_{3} \leq & -k_{s} r^{2}-\theta_{0} \tilde{z}^{2}-\zeta_{0} \frac{\theta_{0}}{\theta_{1}}\left(\tilde{z}-\zeta_{0}\right)^{2}-\frac{\theta_{0}}{\theta_{1}} \sum_{k=1}^{m} \sigma_{k}\left(\tilde{z}-\zeta_{k}\right)^{2} \\
& +\left[-\theta_{1} \tilde{f}(\dot{q}) z r-k_{n}\left(\theta_{1} \dot{q}\right)^{2} r^{2}\right]
\end{aligned}
$$

which can be upperbounded in the following manner

$$
\dot{V}_{3} \leq-\varepsilon_{3}\|\mathbf{y}\|^{2}+\left[\rho \delta_{0}\left(\theta_{1}|\dot{q}|\right) r-k_{n}\left(\theta_{1} \dot{q}\right)^{2} r^{2}\right]
$$

where the positive constant parameter $\varepsilon_{3}$, is given by

$$
\varepsilon_{3}=\min \left\{k_{s}, \theta_{0}, \sigma_{0} \frac{\theta_{0}}{\theta_{1}}, \sigma_{1} \frac{\theta_{0}}{\theta_{1}}, \ldots, \sigma_{m} \frac{\theta_{0}}{\theta_{1}}\right\}
$$

and we have utilized (67) and the fact that the internal friction state $z(t)$ is bounded according to (8). After ap- 
plying the nonlinear damping argument [18] to the bracketed terms of (81), we obtain the following upperbound for $\dot{V}_{3}(t)$

$$
\dot{V}_{3} \leq-\varepsilon_{3}\|\mathbf{y}\|^{2}+\frac{\left(\rho \delta_{o}\right)^{2}}{k_{n}}
$$

which can be further upper bounded as follows

$$
\dot{V}_{3} \leq-\frac{\varepsilon_{3}}{\varepsilon_{2}} V_{3}+\frac{\varepsilon_{3}}{2 \varepsilon_{2}} \frac{1}{\gamma_{0}} \tilde{\sigma}_{0}^{2}+\frac{\varepsilon_{3}}{2 \varepsilon_{2}} \sum_{k=1}^{m} \frac{1}{\gamma_{k}} \tilde{\sigma}_{k}^{2}+\frac{\left(\rho \delta_{o}\right)^{2}}{k_{n}}
$$

where (78) has been utilized. Since the projection algorithm of (70) guarantees $\tilde{\sigma}_{0}(t), \tilde{\sigma}_{k}(t), \hat{\sigma}_{0}(t), \hat{\sigma}_{k}(t) \in L_{\infty}$, we can use (83) and the fact that $V_{3}(t) \geq 0$ to show that $V_{3}(t) \in L_{\infty}$; hence, from (78), we now know that $\mathbf{y}(t) \in L_{\infty}$. Standard signal chasing arguments can be used to show that all signals in the controller/observer and the mechanical system remain bounded during closed-loop operation.

After integrating both sides of (82) and rearranging the terms in a beneficial manner, we obtain

$$
\begin{aligned}
\int_{0}^{T}\|\mathbf{y}(\xi)\|^{2} d \xi & \leq \frac{V_{3}(0)-V_{3}(T)}{\varepsilon_{3}}+\frac{\left(\rho \delta_{0}\right)^{2} T}{\varepsilon_{3} k_{n}} \\
& \leq \frac{V_{3}(0)}{\varepsilon_{3}}+\frac{\left(\rho \delta_{0}\right)^{2} T}{\varepsilon_{3} k_{n}}
\end{aligned}
$$

where $T$ denotes an arbitrary limit of integration, and we have taken advantage of the fact that $V_{3}(t) \geq 0$. After multiplying through by $\frac{1}{T}$ and performing a limit operation to both sides of (84), we obtain following upperbound for the average norm of the composite vector $\mathbf{y}(t)$

$$
\lim _{T \rightarrow \infty} \frac{1}{T} \int_{0}^{T}\|y(\xi)\|^{2} d \xi \leq \frac{\left(\rho \delta_{0}\right)^{2}}{\varepsilon_{3} k_{n}}
$$

Hence, from the definition of (79) and (85), the average square integral of the filtered position tracking error $r(t)$ can be upper bounded as

$$
\lim _{T \rightarrow \infty} \frac{1}{T} \int_{0}^{T} r^{2}(\xi) d \xi \leq \frac{\left(\rho \delta_{0}\right)^{2}}{\varepsilon_{3} k_{n}}
$$

It is clear from (86) that the average square integral of the filtered position tracking error can be arbitrarily small by increasing the nonlinear damping $k_{n}$ or decreasing the constant $\rho$ (i.e., the magnitude $\rho$ will be smaller if we select larger values for $m$ in the approximating function given in (63). Since the filtered tracking error is related to the position tracking error through the linear relationship given by (10), it is an easy matter to calculate an average square integral relationship for the position tracking error similar to that given by (86).

\section{EXPERIMENTAL RESULTS}

A test-bed consisting of a 2-link, direct-drive robot manipulator manufactured by Integrated Motion Inc. was used to implement the observer-based exact model knowledge controller of Section IV and the adaptive controller of Section V. The links of the manipulator are actuated by NSK switched-reluctance motors (39.2 Nm Model RS0608 and 245.0 Nm Model RS-1410) which are controlled through custom NSK power electronics operating in torque control mode. Two integrally mounted resolvers provide rotor position measurements with each resolver having a resolution of 0.00234 degrees. A Pentium $266 \mathrm{MHz}$ PC operating under QNX hosts the control algorithm, which was implemented via Qmotor 2.0, an in-house graphical user-interface, to facilitate real-time graphing, data logging, and the ability to adjust control gains without recompiling the program (for further information on Qmotor 2.0 the reader is referred to [11]). Data acquisition and control implementation were performed at a frequency of $2.0 \mathrm{KHz}$ using the Quanser MultiQ I/O board.

The dynamics for the IMI robot manipulator operating in nonlinear dynamic friction effects are given as follows [16]

$$
\begin{aligned}
{\left[\begin{array}{l}
\tau_{1} \\
\tau_{2}
\end{array}\right]=} & \underbrace{\left[\begin{array}{cc}
m_{1}+2 m_{3} \cos \left(q_{2}\right) & m_{2}+m_{3} \cos \left(q_{2}\right) \\
m_{2}+m_{3} \cos \left(q_{2}\right) & m_{2}
\end{array}\right]\left[\begin{array}{c}
\ddot{q}_{1} \\
\ddot{q}_{2}
\end{array}\right]}_{M(q) \ddot{q}} \\
+ & \underbrace{\left[\begin{array}{cc}
-m_{3} \sin \left(q_{2}\right) \dot{q}_{2} & -m_{3} \sin \left(q_{2}\right)\left(\dot{q}_{1}+\dot{q}_{2}\right) \\
m_{3} \sin \left(q_{2}\right) \dot{q}_{1} & 0
\end{array}\right]}_{V_{m}(q, \dot{q}) \dot{q}} \\
+ & F_{\tau}
\end{aligned}
$$

where $m_{1}=3.31 \mathrm{~kg} \cdot \mathrm{m}^{2}, m_{2}=0.116 \mathrm{~kg} \cdot \mathrm{m}^{2}, m_{13}=0.16 \mathrm{~kg} \cdot \mathrm{m}^{2}$, and the friction torque $F_{\tau}(t) \in \mathfrak{R}^{2 \times 1}$ is explicitly given as

$$
\begin{aligned}
& F_{\tau}=\left[\begin{array}{cc}
f_{d 1} & 0 \\
0 & f_{d 2}
\end{array}\right]\left[\begin{array}{l}
\dot{q}_{1} \\
\dot{q}_{2}
\end{array}\right]+\left[\begin{array}{cc}
\theta_{01} & 0 \\
0 & \theta_{02}
\end{array}\right]\left[\begin{array}{l}
z_{1} \\
z_{2}
\end{array}\right] \\
&-\left[\begin{array}{cc}
\theta_{11} & 0 \\
0 & \theta_{12}
\end{array}\right]\left[\begin{array}{cc}
\frac{\left|\dot{q}_{1}\right|}{\beta_{01}+\beta_{11} \exp \left(-\frac{\dot{q}_{1}^{2}}{\beta_{21}}\right)} \\
0 & \frac{\left|\dot{q}_{2}\right|}{\beta_{02}+\beta_{12} \exp \left(-\frac{\dot{q}_{2}^{2}}{\beta_{22}}\right)}
\end{array}\right]\left[\begin{array}{l}
z_{1} \\
z_{2}
\end{array}\right]
\end{aligned}
$$


with $f_{d 1}=2.0 \mathrm{Nm} \cdot \mathrm{sec}, f_{d 2}=0.5 \mathrm{Nm} \cdot \mathrm{sec}, \theta_{01}=12.0 \mathrm{Nm}$, $\theta_{02}=5.0 \mathrm{Nm}, \theta_{11}=0.2 \mathrm{Nm} \cdot \mathrm{sec}, \theta_{12}=0.2 \mathrm{Nm} \cdot \mathrm{sec}, \beta_{01}=$ $0.84, \beta_{11}=0.125, \beta_{21}=0.001, \beta_{02}=0.54, \beta_{12}=0.06$, and $\beta_{22}=0.002$.

Remark 7. The system parameters associated with (88) were calculated using the following procedure: First, a sinusoidal torque input with the peak amplitude selected to be more than the static friction torque was applied to each actuator. The actuator acceleration for each link was computed by applying a backwards difference algorithm to the corresponding measured velocity signal and then filtering the resulting signal. The experimentally obtained frictional torque $F_{\tau}(t)$ was then calculated by subtracting the inertial torque $M(q) \ddot{q}$ and the torque associated with the centripetal-Coriolis terms $V_{m}(q, \dot{q}) \dot{q}$ from the applied torque $\tau(t)$ of (87). The various parameters of (88) were then adjusted to fit the experimentally obtained profile of the friction torque.

\section{Experiment 1}

In this experiment, the desired link trajectories (see Fig. 1 and Fig. 2) were selected as follows

$$
\left[\begin{array}{l}
q_{d 1} \\
q_{d 2}
\end{array}\right]=\left[\begin{array}{l}
45.0 \tan ^{-1}(3.0 \sin (0.5 t))\left(1-\exp \left(-0.01 t^{3}\right)\right) \\
45.0 \tan ^{-1}(3.0 \sin (0.4 t))\left(1-\exp \left(-0.01 t^{3}\right)\right)
\end{array}\right](\operatorname{deg}) .
$$

The observer/controller scheme of (15), (17), and (18) was implemented with the following control gain values (note: the control gains were selected in a trial and error fashion to minimize the link position tracking error)

$$
k_{s 11}=19.0 \quad k_{s 22}=4.0 \quad \alpha_{11}=125.5 \quad \alpha_{22}=158.0
$$

The position tracking error for each link is shown in Fig. 1 and Fig. 2.

Remark 8. In the above implementation, the rotor velocity signal is obtained by applying a standard backwards difference algorithm to the position signal with the resulting signal being filtered by a second-order digital filter. In addition, the integral structure of the observer schemes, the adaptation laws, and the nonlinear filters were computed on-line via a standard trapezoidal algorithm. The control gains of (90) were determined through a trial and error procedure to minimize the position tracking error.

\section{Experiment 2}

For this experiment, the desired link trajectories of (89) were again utilized (see Fig. 1 and Fig. 2). The observer/controller scheme of (31), (32), (33), and (35) was implemented with the following gain values (note: the
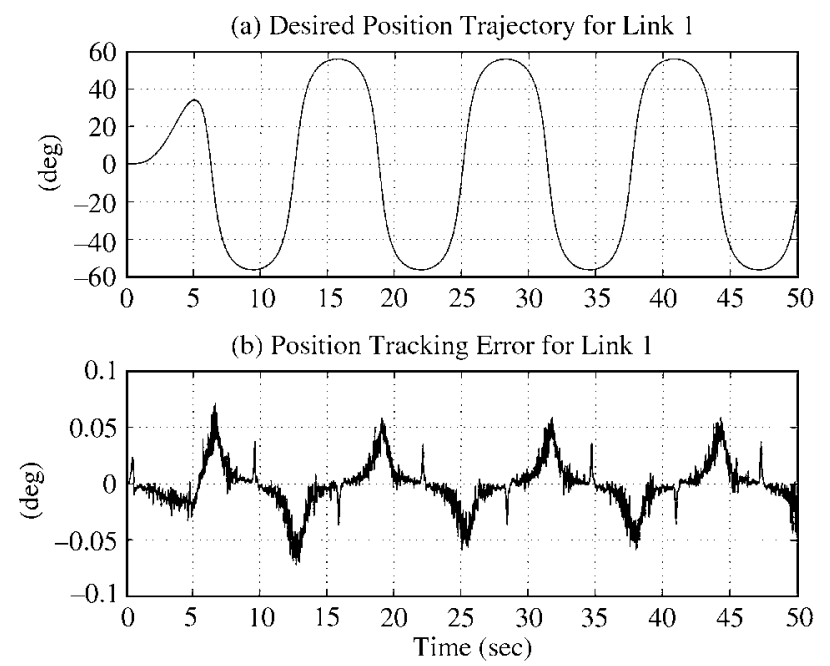

Fig. 1. (a) Desired position trajectory for link 1 (b) Position tracking error for link 1 .

(a) Desired Position Trajectory for Link 2

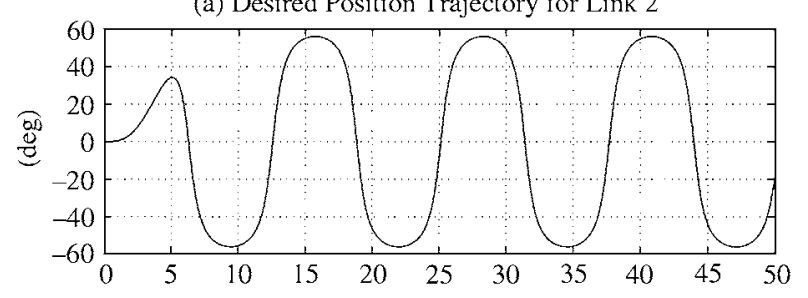

(b) Position Tracking Error for Link 2

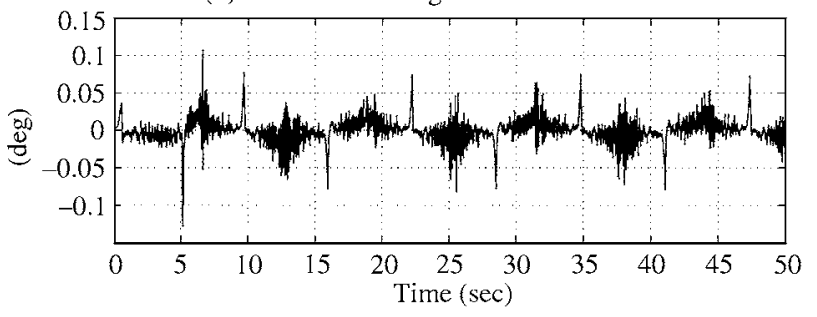

Fig. 2. (a) Desired position trajectory for link 2 (b) Position tracking error for link 2 .

control/adaptive gains are selected in a trial and error manner to achieve the best link position tracking error)

$$
\begin{aligned}
& k_{s 11}=84.0 \quad k_{s 22}=36.5 \quad \alpha_{11}=70.0 \quad \alpha_{22}=46.0 \\
& \gamma_{01}=0.3 \quad \gamma_{02}=0.65 \quad \gamma_{11}=0.001 \quad \gamma_{12}=0.005 \\
& \Gamma_{m}=\text { diag }\{0.95,0.3,0.5,12.75,6.5\} .
\end{aligned}
$$

All parameter estimates and auxiliary filter states are initialized to zero. The corresponding link position tracking error is shown in Fig. 3. The various adaptive estimates are shown in Figs. 4, 5, 6 and 7.

Remark 9. The observer-based adaptive controller of (31), (32), (33), and (35) was initially tuned with all adaptation gains set to zero (note that the parameter estimates are zero 
(a) Position Tracking Error for Link 1

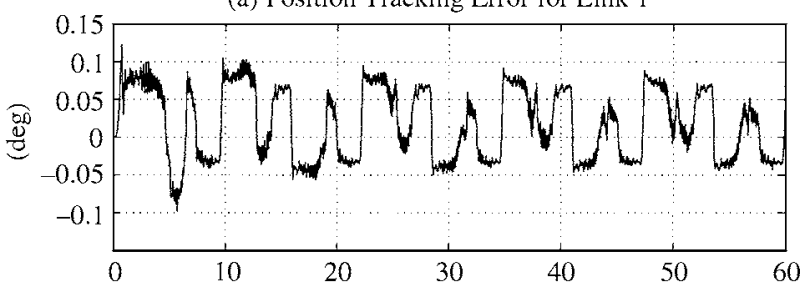

(b) Position Tracking Error for Link 2

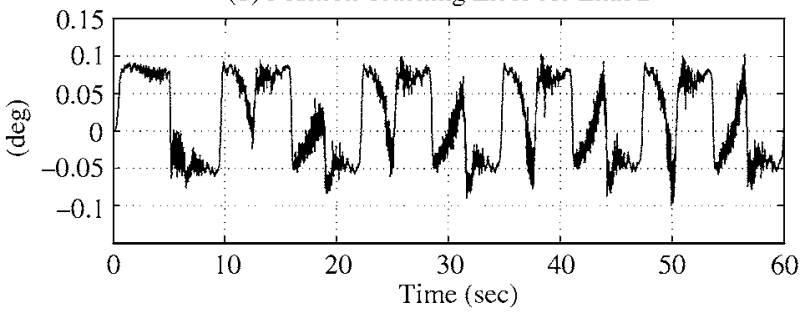

Fig. 3. (a) Position tracking error for link 1 (b) Position tracking error for link 2.

(a) Parameter Estimate for $\mathrm{m}$

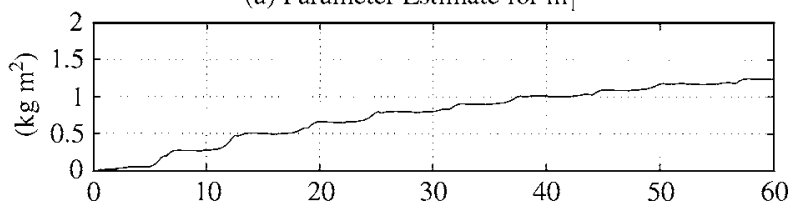

(b) Parameter Estimate for $m_{2}$

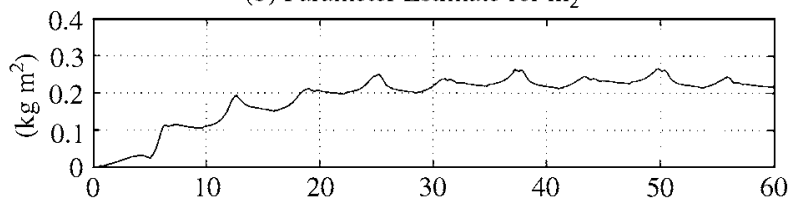

(c) Parameter Estimate for $m_{3}$

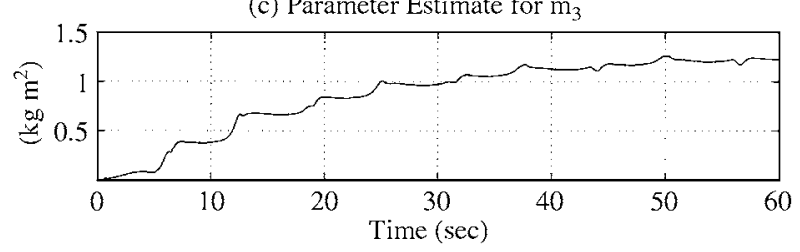

Fig. 4. (a) $\hat{m}_{1}(t)\left(\right.$ b) $\hat{m}_{2}(t)$ (c) $\hat{m}_{3}(t)$.

during this procedure). That is, the control gains were adjusted to minimize the position tracking error. At some point, significant improvement in the position tracking error could not be attained by adjustments of only the control gains. The adaptation gains were then adjusted to allow the parameter estimation to reduce the position error to that shown in Fig. 3.

\section{Experiment 3}

To test the performance of the observer/controller algorithm of Section VI, a one degree of freedom experimental test-bed was utilized (see Fig. 8). It consisted of a $88 \mathrm{Nm}$ switched reluctance motor (NSK Corp., Model RS0810) operating in torque control mode. A metal disk (a) Parameter Estimate for $f_{d}$

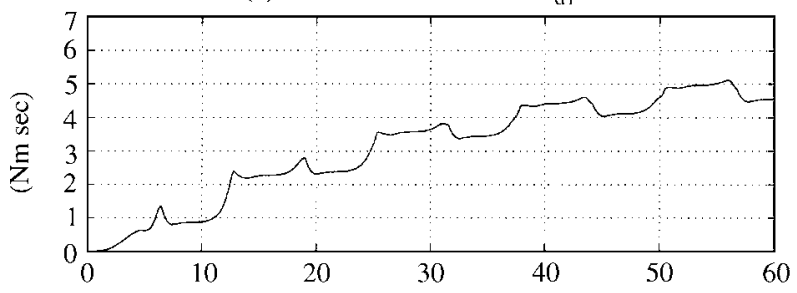

(b) Parameter Estimate for $\mathrm{f}_{\mathrm{d} 2}$

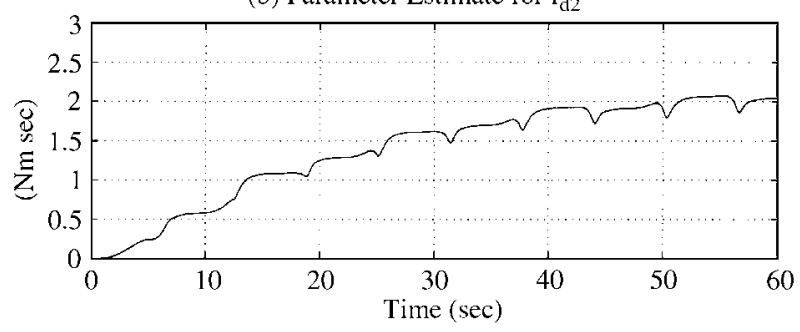

Fig. 5. (a) $\hat{f}_{d 1}(t)$ (b) $\hat{f}_{d 2}(t)$.

(a) Parameter Estimate for $\theta_{01}$

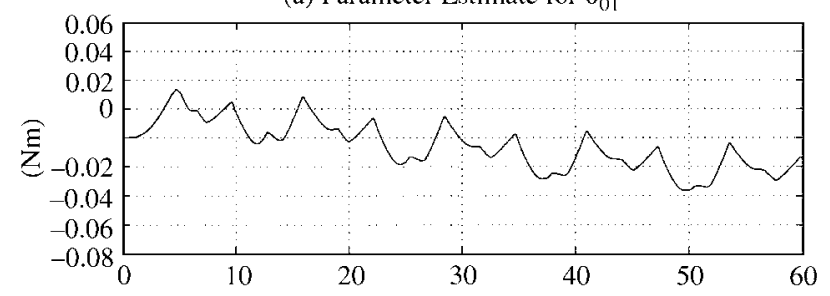

(b) Parameter Estimate for $\theta_{02}$

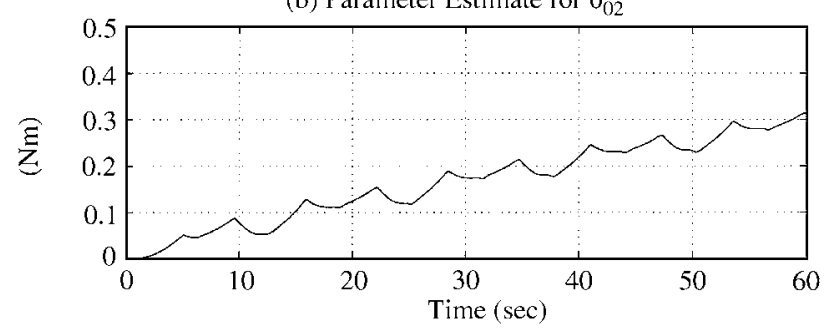

Fig. 6. (a) $\hat{\theta}_{01}(t)$ (b) $\hat{\theta}_{02}(t)$.
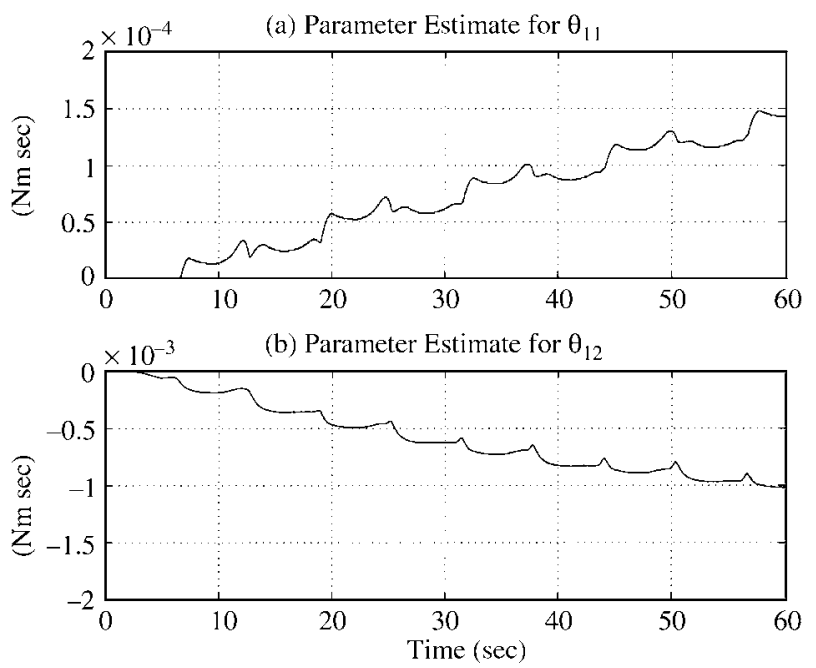

Fig. 7. (a) $\hat{\theta}_{11}(t)$ (b) $\hat{\theta}_{12}(t)$. 


\section{Mechanical System}

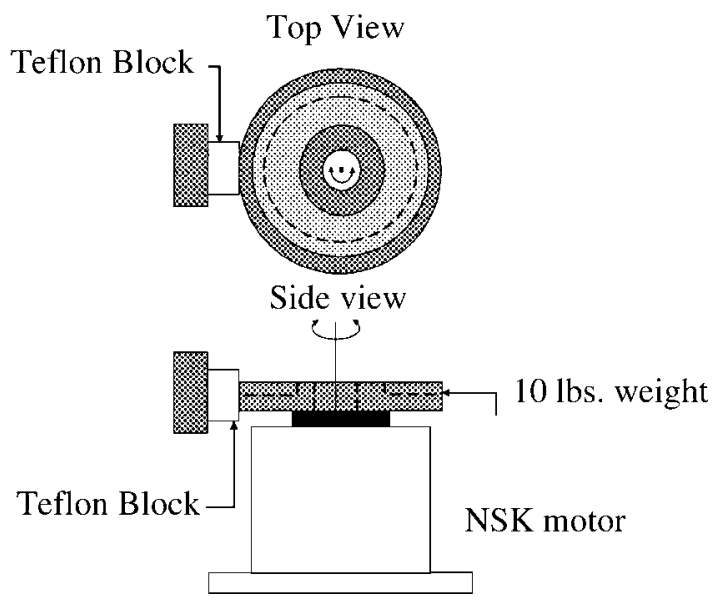

Fig. 8. Schematic of experimental setup.

weighing $11 \mathrm{lbs}$. is attached to the rotor concentrically. Friction is introduced via a block of Teflon ${ }^{\mathrm{R}}$ held against the disk as shown in the Fig. 8. The amount of friction can be varied by adjusting a vice arrangement. An integrally mounted resolver provides rotor position measurements with a resolution of 0.00234 degrees. A QNX based realtime Photon environment developed in-house serves as the user-interface required to implement the control algorithm. The control algorithm was computed on a $200 \mathrm{MHz}$ Pentium processor. The sampling frequency was selected to be $3000 \mathrm{~Hz}$. The MultiQ board (8 A/D, 8 D/A, and 6 encoder channels) manufactured by Quanser Consulting was used to output the desired torque signal and read in the quadrature position related signal.

For the model given by (59) and (60), the numerical values of the system parameters were determined to $b^{6}$

$$
\begin{array}{rlrl}
M & =0.125 \mathrm{~kg} \cdot \mathrm{m}^{2} & B=0.5 \mathrm{Nm} \cdot \mathrm{sec} & \theta_{0}=8.0 \mathrm{Nm} \\
\theta_{1}=0.1 \mathrm{Nm} \cdot \mathrm{sec} & \beta_{0}=1.06 & \beta_{1}=0.06 \\
\beta_{2}=2.24 & &
\end{array}
$$

For this experiment, the desired position trajectory $q_{d}$ $(t)$ was selected as

$$
q_{d}=90.0 \tan ^{-1}(4.0 \sin (0.5 t))\left(1-\exp \left(-0.01 t^{3}\right)\right)(\mathrm{deg}) .
$$

The selected number of approximating functions and the values for the adaptation/damping/control gains ${ }^{7}$ achieving the best link position tracking performance are given as follows

\footnotetext{
${ }^{6}$ The system parameters of (92) were determined in a similar manner as outlined in Remark 7.

${ }^{7}$ The adaptation/control gains of (94) were determined through an identical strategy as given in Remark 9.
}

$$
m=21 \quad \alpha=45.0 \quad k_{s}=10.0 \quad \gamma=10.0 \quad k_{n}=1.0
$$

where we have substituted the positive scalar constant $\gamma$ for all adaptation gain values (i.e., $\gamma_{0}=\gamma_{k^{\prime} s}=\gamma$ ). All initial parameter estimates and initial filter states are set to zero. The resulting position tracking error is shown in Fig. 9. The parameter estimates for three of the selected weighting coefficients are shown in Fig. 10.

Remark 10. From the selection of $m$ in (94), the implementation of the controller requires the on-line calculation of 22 dynamic weighting coefficient estimates, 22 auxiliary filter variables, in addition to the calculation of the observer variable $\hat{z}(t)$; hence, the maximum value for $m$ is constrained by the selected sampling frequency.

(a) Desired Position Trajectory

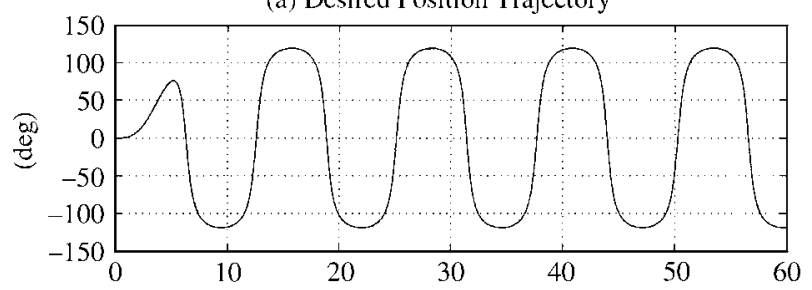

(b) Position Tracking Error

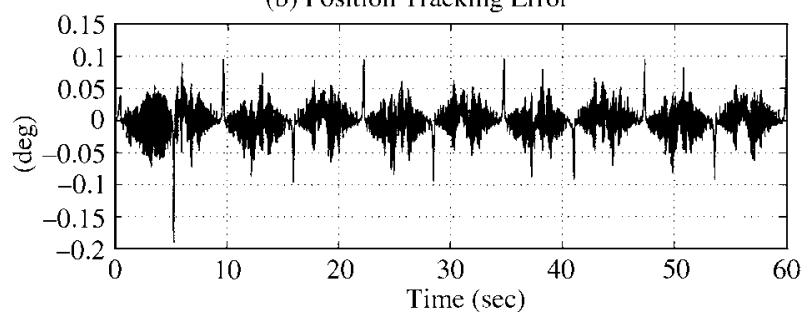

Fig. 9. (a) Desired position trajectory (b) Position tracking error.

(a) Parameter Estimate for $\sigma_{0}$

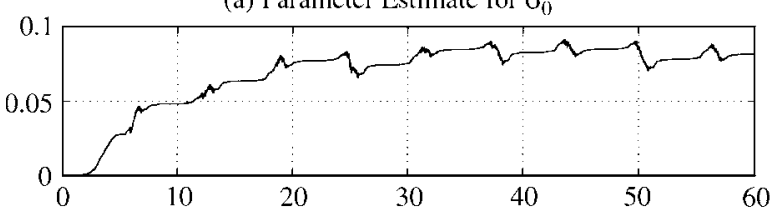

(b) Parameter Estimate for $\sigma_{10}$

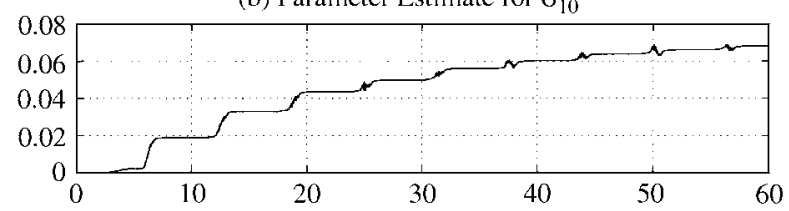

(c) Parameter Estimate for $\sigma_{21}$

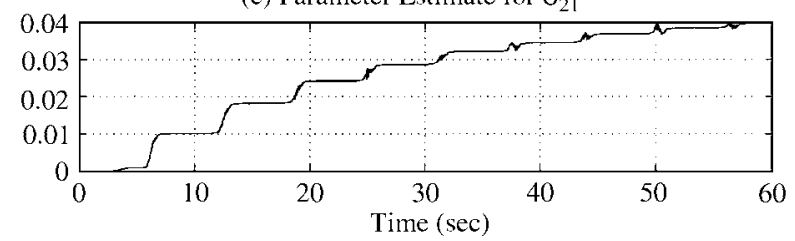

Fig. 10. (a) $\hat{\sigma}_{0}(t)(\mathrm{b}) \hat{\sigma}_{10}(t)(\mathrm{c}) \hat{\sigma}_{21}(t)$ 


\section{Discussion}

In Experiment 1, we observe approximately \pm 0.05 degrees of tracking error for link 1 and \pm 0.08 degrees of tracking error for link 2 from Figs. 1 and 2, respectively, for identical desired position trajectories in (89). From Fig. 1, we see that the maximum magnitude of position tracking error for link 1 occurs at large link velocities (i.e., during the transition period of the sinusoidal trajectory). This phenomenon could indicate a possible miscalculation of the viscous friction parameter $f_{d 1}$ in (88) from the procedure outlined in Remark 7; hence, the inaccuracy in $f_{d 1}$ is then relayed to the feed-forward cancellation term $F_{d} \dot{q}$ of (14) in the exact model knowledge controller of Section IV which may attribute to the relatively minor degradation in tracking performance. In contrast, we see that the maximum magnitude of the tracking error for link 2 coincides with the peaks of the sinusoidally shaped position profiles (i.e., when the link velocity is close to zero). Such aspects as link velocity measurements and parameter mismatch may contribute to the somewhat small peaks observed in Fig. 2. Experiment 2 illustrates the attractiveness of the adaptive controller of Section 5.1 by obtaining approximately the same order of magnitude of position tracking error $( \pm 0.08$ degrees and \pm 0.09 degrees for links 1 and 2 , respectively) as the exact model knowledge controller of Section IV without a priori knowledge of selected system parameters. Specifically, the adaptive controller implemented in Experiment 2 requires only the knowledge of the parameters associated with the Stribeck function $f(\dot{q})$ (i.e, the $\beta_{0 i^{\prime} s}$ and $\beta_{l^{\prime} s}$ of (88)). With the construction of the scalar mechanical system shown in Fig. 8, we were able to introduce exaggerated friction levels as compared to the assembled IMI robot manipulator in Experiment 3. That is, in order to better observe the level of performance of the proposed adaptive controller of Section VI, the vice arrangement was adjusted in a manner to create relatively high friction effects. From Fig. 9, an approximate steady state position tracking error of \pm 0.09 degrees is obtained. As commented in Remark 10, the level of the position tracking error achieved is dependent on the selection of the number of approximating functions $m$ of (63). However, we note that increasing the value of $m$ increases the order of the overall control algorithm (i.e., the increase in the value of $m$ by one introduces an additional parameter estimate accompanied with an additional filter state). Hence, with the increase in the value of $m$, the computational burden/complexity of implementing the control algorithm is consequently increased. We note that the value of $m$ in (94) was selected through a trial and error procedure. Specifically, the function $f(\dot{q})$ of (62) was first simulated utilizing the parameter values of (92) over a range over a range of velocities $\dot{q}(t)$. The approximated function $\bar{f}(\dot{q})$ was then plotted over the same range of velocities for various values $m$ and weighting parameters $\sigma_{i^{\prime} s}$ until a suitable curve-fit was obtained (i.e., the value of $m$ determined was then used in Experiment 3 and the determined values for weighting parameters were discarded). Currently, there do not exist established guidelines for the selection of $m$. Typically, a trial and error procedure will be employed to determine the value of $m$ based on the tracking error performance under the constraint of sampling frequency (i.e., the control routine must be able to calculate the parameter estimates of (70) in addition to the filter variables of (71) within the given sampling interval).

For the sake of brevity, a detailed comparison between various friction models was not performed. For a comparison between the standard (static) friction model [12] and the Lu-Gre model (dynamic) of friction [7], we refer the reader to the previous work of Vedagarbha et al. [25].

\section{CONCLUSIONS}

In this paper, we have illustrated how the previously published observer/controller schemes of [25] can be extended to the $n$-link, serially connected, direct drive, rigid-link, revolute robot. Specifically, we developed a partial-state feedback observer/controller scheme to achieve global exponential link position tracking. In addition, we have developed two adaptive controllers that compensated for selected parametric uncertainty and achieved global asymptotic link position tracking. We have also presented a new adaptive control technique that compensated for the nonlinear parameterizable Stribeck effects and forced the average square integral of the position tracking error to an arbitrarily small value. Experimental results were presented to illustrate the ability of the controllers to compensate for friction effects. Future work will involve the development of a control strategy that can adaptively compensate for parametric uncertainty throughout the entire mechanical model.

\section{REFERENCES}

1. Annaswamy, A., F., Skantze and A. Loh, "Adaptive Control of Continuous Time Systems with Convex/ Concave Parametrization," Automatica, Vol. 34, No. 1, pp. 33-49 (1998).

2. Armstrong-Hélouvry, B., "Stick-slip Arising from Stribeck Friction," Proc. the IEEE Int. Conf. Rob. Autom., Cincinnati, OH, pp. 1377-1382 (1990).

3. Armstrong-Hélouvry, B., "A Survey of Models, Analysis Tools and Compensation Methods for the Control of Machines with Friction," Automatica, Vol. 30, No. 7, pp. 1083-1138 (1994).

4. Armstrong-Hélouvry, B., "Stick-Slip and Control in Low-Speed Motion," IEEE Trans. Automat. Contr., Vol. 38, No. 10, pp. 1483-1496 (1993).

5. Bliman, P.A. and M. Sorine,"Easy-to-use Realistic Dry Friction Models for Automatic Control," Proc. the Eur. Contr. Conf., Roma, Italy, pp. 3788-3794 (1995). 
6. Bridges, M.M., D. Dawson and C.A. Abdallah, "Control of Rigid-Link Flexible-Joint Robots: A Survey of Backstepping Approaches," J. Rob. Syst., Vol. 12, No. 3, pp. 199-216 (1995).

7. Canudas de Wit, C., H. Olsson, K.J. Åström and P. Lichinsky, "A New Model for Control of Systems with Friction," IEEE Trans. Automat. Contr., Vol. 40, No. 3, pp. 419-425 (1995).

8. Canudas de Wit, C., P. Noel, A. Aubin and B. Brogliato, "Adaptive Friction Compensation in Robotic Manipulators: Low Velocities," Int. J. Rob. Res., Vol. 10, No. 3, pp. 189-199 (1991).

9. Canudas de Wit, C. and P. Lichinsky, "Adaptive Friction Compensation with Dynamic Friction Model," Proc. the IFAC World Cong., San Francisco, CA, pp. 197-202 (1996).

10. Canudas de Wit, C. and R. Kelly, "Passivity-based Control Design for Robots with Dynamic Friction," Proc. the IASTED Int. Conf. Rob. Manuf., Cancun, Mexico, pp. 84-87 (1997).

11. Costescu, N., D.M. Dawson and M. Loffler, "QMotor 2.0 - A Real-Time PC Based Control Environment," IEEE Contr. Syst. Mag., Vol. 19, No. 3, pp. 68-76 (1999).

12. Feemster, M., P. Vedagarbha, D.M. Dawson and D. Haste, "Adaptive Control Techniques for Friction Compensation," Mechatronics - An Int. J., Vol. 9, No. 2, pp. 125-145 (1999).

13. Friedland, B. and Y.J. Park, "On Adaptive Friction Compensation," IEEE Trans. Automat. Contr., Vol. 37, No. 10, pp. 1609-1612 (1992).

14. Gonzalez, J. and G. Widmann, “A New Model for Nonlinear Friction Compensation in the Force Control of Robot Manipulators," Proc. the IEEE Conf. Contr. Appl., Hartford, CT, pp. 201-203 (1997).

15. Haessig, D.A. and B. Friedland, "On the Modeling and Simulation of Friction," J. Dyn. Syst., Meas. Contr., Vol. 113, No. 3, pp. 354-362 (1991).

16. Direct Drive Manipulator Research and Development Package Operations Manual, Integrated Motion Inc., Berkeley, CA (1992).

17. Jain, S., F. Khorrami, N. Ahmad and S. Sankaranarayanan, "Friction Compensation for Drives with and without Transmission Compliance," Proc. the IEEE Amer. Contr. Conf., Albuquerque, NM, pp. 2925-2929 (1997).

18. Kokotovic, P., "The Joy of Feedback: Nonlinear and Adaptive," IEEE Contr. Syst. Mag., Vol. 12, No. 3, pp. 7-17 (1992).

19. Lischinsky, P., Canudas de Wit C. and G. Morel, "Friction Compensation of a Schilling Hydraulic Robot," Proc. the IEEE Conf. Contr. Appl., Hartford, CT., pp. 294-299 (1997).

20. Olsson, H. and K. Astrom, "Observer-based Friction Compensation,” Proc. IEEE Conf. Decis. Contr., Kobe, Japan, pp. 4345-4350 (1996).
21. Panteley, E., R. Ortega and M. Gafvert, "An Adaptive Friction Compensator for Global Tracking in Robot Manipulators," Syst. \& Contr. Lett., Vol. 33, No. 5, pp. 307-313 (1998).

22. Sastry, S. and M. Bodson, Adaptive Control Stability, Convergence, and Robustness, Prentice Hall, Englewood Cliffs, New Jersey (1989).

23. Sivakumar, S. and F. Khorrami, "Friction Compensation via Variable Structure Control: Regulation and Low Velocity Tracking," Proc. the IEEE Conf. Contr. Appl., Hartford, CT., pp. 645-650 (1997).

24. Slotine, J.J.E. and W. Li, Applied Nonlinear Control, Prentice Hall, Englewood Cliffs, New Jersey (1991).

25. Vedagarbha, P., D.M. Dawson and M. Feemster, "Tracking Control of Mechanical Systems in the Presence of Nonlinear Dynamic Friction Effects," IEEE Trans. Contr. Syst. Technol., Vol.7, No.4, pp. 446-456 (1999).

26. Yazdizadeh, A. and K. Khorasani, "Adaptive Friction Compensation using a Lyapunov-Based Design Scheme," Proc. the IEEE Conf. Decis. Contr., Kobe, Japan, pp. 2830-2831 (1996).

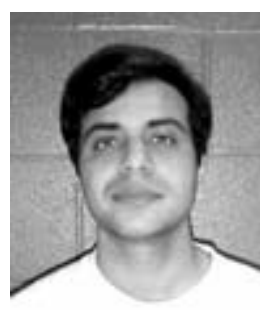

Matthew Feemster received the B.S degree in Electrical Engineering from Clemson University, Clemson, South Carolina in December 1994. Upon graduation, he remained at Clemson University and received the M.S. degree in Electrical Engineering in 1997. During this time, he also served as a research/teaching assistant. His research work focused on the design and implementation of various nonlinear control algorithms with emphasis on the induction motor and mechanical systems with friction present. He is currently working toward his Ph.D. degree in Electrical Engineering at Clemson University.

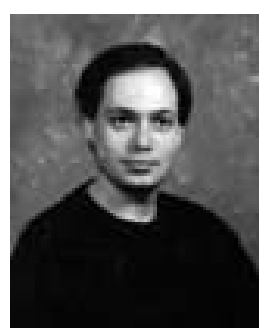

Darren M. Dawson was born in 1962 , in Macon, Georgia. He received an Associate Degree in Mathematics from Macon Junior College in 1982 and a B.S. Degree in Electrical Engineering from the Georgia Institute of Technology in 1984. He then worked for Westinghouse as a control engineer from 1985 to 1987 . In 1987, he returned to the Georgia Institute of Technology where he received the Ph.D. Degree in Electrical Engineering in March 1990. During this time, he also served as a research/teaching assistant. In July 1990, he joined the Electrical and Computer Engineering Department and the Center for Advanced Manufacturing 
(CAM) at Clemson University where he currently holds the position of Professor. Under the CAM director's supervision, he currently leads the Robotics and Manufacturing Automation Laboratory which is jointly operated by the Electrical and Mechanical Engineering departments. His main research interests are in the fields of nonlinear based robust, adaptive, and learning control with application to electromechanical systems including robot manipulators, motor drives, magnetic bearings, flexible cables, flexible beams, and high-speed transport systems.

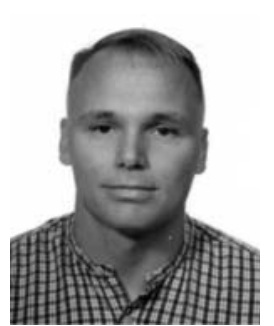

Aman Behal was born in India in 1973. He received his Masters Degree in Electrical Engineering from Indian Institute of Technology, Bombay in 1996. He is currently working towards a Ph.D in Controls and Robotics at Clemson University. His research focuses on the control of nonlinear systems with special interest in motor control and underactuated systems.

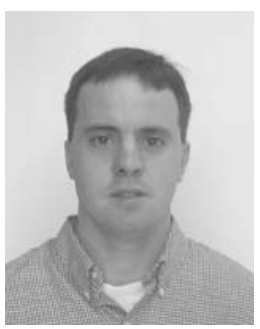

Warren Dixon was born in York, Pennsylvannia on June 15th, 1972. He recieved the Bachelor of Science degree in 1994 from the Department of Electrical and Computer Engineering from Clemson University, Clemson, South Carolina. He then recieved the Master of Engineering degree in 1997 from the Department of Electrical and Computer Engineering from the University of South Carolina, Columbia, South Carolina. He returned to Clemson University where he is currently pursuing a Ph.D. degree. As a student in the Robotics and Mechatronics group at Clemson University, his research is focused on nonlinear based robust and adaptive control techniques with application to electromechanical systems including wheeled mobile robots, twin rotor helicopters, surface vessels, and robot manipulators. 